International Journal of Applied Mathematical Research, 9(1)(2019) $21-31$
International Journal of Applied Mathematical Research
SPC
Website: www.sciencepubco.com/index.php/IJAMR
Research paper

\title{
Stability analysis of a mathematical model for awareness initiatives on registration of persons in Kenya
}

\author{
Ann N. Mwambia ${ }^{1}$, Mark O. Okongo ${ }^{1}$, Gladys G. Njoroge ${ }^{1}$ \\ ${ }^{1}$ Chuka University, Kenya \\ *Corresponding author E-mail: mwambia.ann@gmail.com
}

\begin{abstract}
In this paper, we discuss stability analysis of a mathematical model of awareness initiatives in registration of persons in Kenya. Using Ordinary Differential Equations, a mathematical model to compare the efficacy of print media, electronic media and word-of-mouth media in disseminating registration information is developed. Positivity and boundedness of solutions is established to ensure that the model is mathematically meaningful. The Basic Reproduction number $\mathrm{R}_{0}$ is derived using the Next Generation Matrix. We present both awareness free equilibrium and the maximum awareness equilibrium. Stability analysis of the model shows that Awareness free equilibrium is both locally and globally asymptotically stable when $\mathrm{R}_{0}<1$ hence no spread of awareness and unstable when $\mathrm{R}_{0}>1$ while MAE is locally asymptotically stable when $\mathrm{R}_{0}>1$ indicating spread of information in the population.
\end{abstract}

Keywords: Aware-Adopters; Awareness Initiatives; Aware-Non-Adopters; Registration of Persons; Reproduction Number; Stability Analysis; Unaware.

\section{Introduction}

Registration of persons is a crucial exercise undertaken by the government for purposes of planning, security and execution of fiscal strategies. Population registration provides a ticket to full enjoyment of rights and freedoms in a country [1]. As such, any delays in the registration and issuance of the identification documents or even issuance of the same to undeserving persons would impact negatively on the individual and the nation at large. Everyone within the borders of Kenya is required to have recognized identification papers. Indeed, one has to be registered either as a Kenyan or a foreigner. Consequently, it is not a matter of choice for one to seek identification documents. The National Registration Bureau (NRB), a department in the Ministry of Interior and Coordination of National Government is mandated by constitution through Registration of Persons Act CAP 107 ( laws of Kenya ) to carry out registration of persons. However, this constitutional right has not been absolutely free of delay-challenges which negatively impact on the individual and the country at large. [2-5] recommended public awareness of the registration requirements as well as importance of ID card as an antidote to these delaychallenges. [2] Report also shows that the value of ID card has been undermined by the fact that its essence is pegged to acquisition of a voter's card, and by extension eligibility to voting in the general election exercises in Kenya. This is evident in crowding of ID cards applicants at the registration offices during electioneering year. The Not Previously Registered (NPR) applications given in Table 1 shows the disproportionate explosion of NPR applicants in the build up to electioneering period.

\begin{tabular}{|c|c|c|c|c|c|c|c|c|c|c|}
\hline YEAR & 2008 & 2009 & 2010 & 2011 & 2012 & 2013 & 2014 & 2015 & 2016 & 2017 \\
\hline NPR Applications & 487348 & 611742 & 930768 & 434162 & 1548942 & 841629 & 913087 & 1097267 & 1529421 & 1233268 \\
\hline
\end{tabular}

According to table 1 the number of ID applications made in 2011, a year before elections was 434162 . The following year which happened to be an election year, the number of NPR applications made ballooned to 1548 942. After elections in early 2013, the number of NPR applications made mysteriously dropped to 841629 .

Table 2: Population Census 2009 (18 Years) and NPR Discrepancy

\begin{tabular}{llll}
\hline Year & Population (Attaining 18 Years) & NPR Applications & Discrepancy \\
\hline 2009 & 887693 & 611742 & 275951 \\
\hline Source: KNBS, Kenya Population and Housing Census 2009. & &
\end{tabular}

Table 2 shows the discrepancy between the section of population who had attained eighteen (18) years in 2009 and those who applied for ID Cards.

There has been numerous terrorism activities in Kenya. The Westgate terrorist attack in Nairobi, extremism activities in Mandera in NorthEastern and Kilifi in coastal region were some examples. NRB cited late registration of persons as a weakness that opens the gap for illegal 
registration, which is exploited by extremist to acquire registration documents [6]. This crowding of ID card applicants at the registration offices during electioneering period strains the NRB personnel and resources. As a result, foreigners take advantage of the crowding and get registered with fake documents and through corruption. Mathematical modelling has become a valuable tool for the analysis of dynamics of infectious diseases and for the support of control strategies in the recent past [7]. Several researchers have developed mathematical models on awareness of the infectious diseases in order to minimize or fully eradicate the disease by various control measures such as use of face masks, quarantine as well as vaccination. In these models, awareness either by media or word of mouth is included whereby it acts on the susceptible and infected population hence reducing the spread of the infection. [8], [9] and [10] formulated models using a system of differential equations. The studies carried out the stability analysis of their respective models and showed that the disease free equilibrium point is locally as well as globally asymptotically stable when $\mathrm{R}_{0}<1$ hence the disease does not spread while the endemic equilibrium point only exists when $\mathrm{R}_{0}>1$ and is locally asymptotically stable, meaning the disease persists. Mathematical models on diseases have formed the basis of mathematically modeling awareness of other phenomena. [11] Studied the influence of awareness programs of media on drinking dynamics. The study showed that alcohol free equilibrium point is locally as well as globally asymptotically stable when $\mathrm{R}_{0}<$ 1 while the alcohol present equilibrium point is locally and globally asymptotically stable when $R_{0}>1$. The results showed that awareness programs reduced the binge drinking. [12] Studied the importance of spreading the awareness information on infant vaccination. The study adopted the Bass model for diffusion of awareness information in ascertaining the most influential medium; between mass media and word of mouth. The study divided the population into three categories namely; unaware, aware - adopters and aware - non-adopters. The study used a system of differential equations to formulate the Model. Stability analysis proved the awareness free equilibrium and maximum awareness equilibrium to be locally and globally asymptotically stable. This paper seeks to modify the model developed by [12] on the importance of spreading the awareness information on infant vaccination, to carry out the stability analysis of the equilibrium points of a mathematical model on awareness initiatives on registration of persons in Kenya. The model developed relates closely in methodology to the awareness creation models of the diseases, vaccination of infants and binge drinking. It divides the media into print, electronic and the word-of-mouth, as opposed to the mathematical models reviewed earlier which mostly deal with media as a whole. The model compartmentalized the population (those attaining 18 years) into three classes; unaware, aware-adopters and aware-non-adopters.

\section{Model formulation}

The study formulates and describes a Mathematical Model for awareness campaigns on the requirements of registration of ID cards and their importance. The model is developed from a system of Ordinary Differential Equations incorporating two stages; awareness stage and a decision making stage. The awareness campaigns is done through Electronic Media, Print Media and Word-of-Mouth. The population (P) which is the number of individuals qualified for ID card registration (those attaining 18 years) and interacting with awareness is divided into three classes: the number of individuals who are not aware of the registration requirements and importance of ID cards (N), the number of individuals who have awareness information on registration of ID cards and have adopted it (M) and the number of individuals who have awareness on registration of ID cards but did not adopt it (A). The study assumes a recruitment rate $\mu$, for the number of individuals who are not aware of registration of ID cards $(\mathrm{N})$. A proportion $\mathrm{q}$ of unaware individuals in class $\mathrm{N}$ is assumed to become aware and adopt registration of ID cards and progress to class $M$ through Electronic Media at a rate $\Omega$ and the remaining proportion become aware but do not adopt and progress to class $\mathrm{A}$ at the same rate. A proportion $\mathrm{r}$ in class $\mathrm{N}$ is assumed to become aware and adopt registration of ID cards and progress to class $\mathrm{M}$ through Print Media at a rate $\phi$ and the remaining proportion become aware but do not adopt and progress to class $\mathrm{A}$ at the same rate. A proportion $\mathrm{s}$ in class $\mathrm{N}$ is assumed to become aware through word- of- mouth and adopt the registration of ID cards and progress to class $\mathrm{M}$ at a rate $\alpha$ and the remaining proportion progresses to class $\mathrm{A}$ at the same rate. Adopters can discontinue from the awareness of registration of ID cards class $\mathrm{M}$ and move to class A at a rate $\psi$. Individuals in non-adopters, class A, can progress to adopters class $\mathrm{M}$ at a rate $\gamma$ or forget the awareness information and return to the class $\mathrm{N}$ at a rate $\theta$. The schematic representation of the Model for the spread of sensitization initiatives of ID cards registration is shown in Figure 1. 


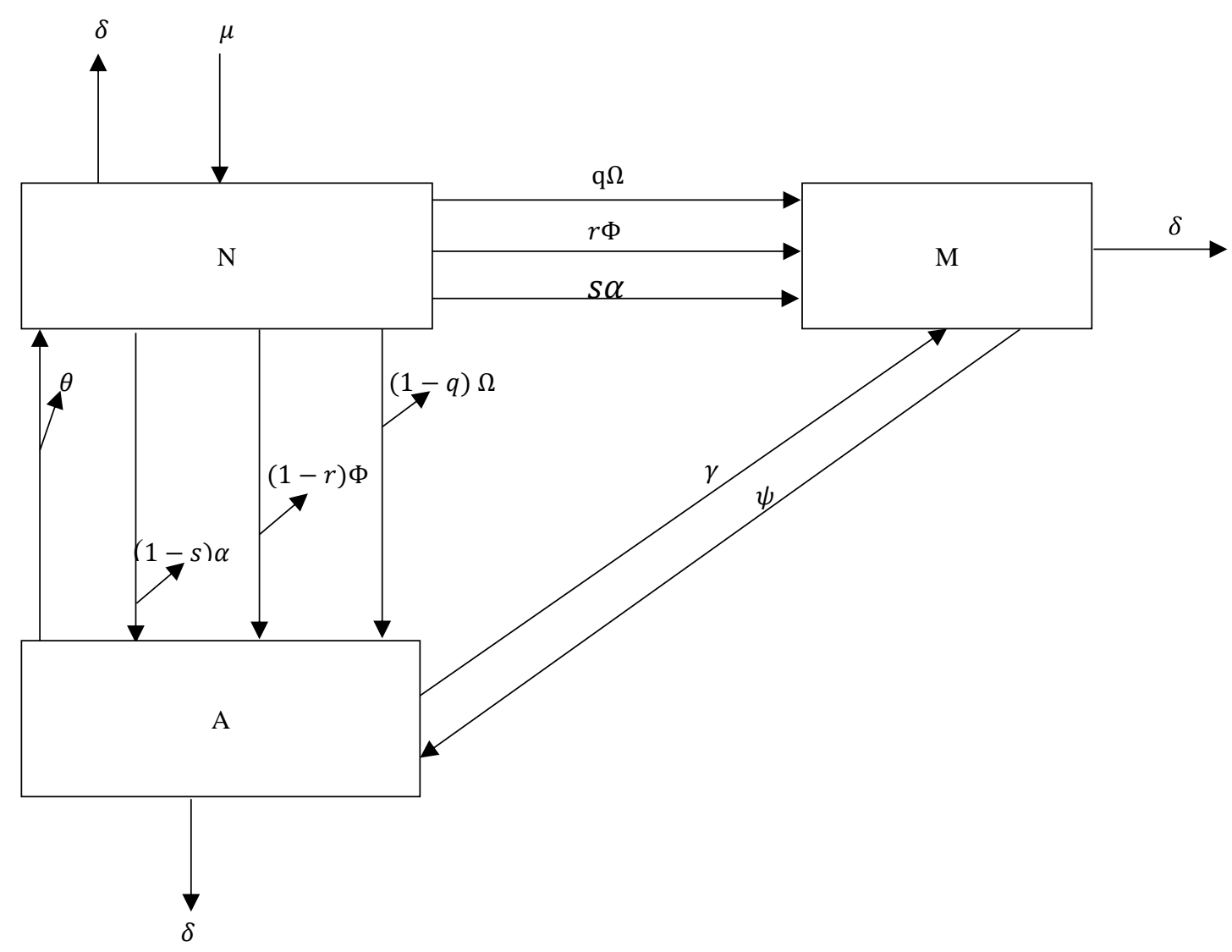

Fig. 1: Diagram of the Model.

The Model can be described by a system of ODEs given below.

$\frac{d N(t)}{d t}=\mu-\Omega N(t)-\phi N(t)-\alpha N(t)\left(\frac{M(t)+A(t)}{P(t)}\right)-\delta N(t)+\theta A(t)$

$\frac{d M(t)}{d t}=q \Omega N(t)+r \phi N(t)+s \alpha N(t)\left(\frac{M(t)+A(t)}{P(t)}\right)-M(t)(\psi+\delta)+\gamma A(t)$

$\frac{d A(t)}{d t}=(1-q) \Omega N(t)+(1-r) \phi N(t)+(1-s) \alpha N(t)\left(\frac{M(t)+A(t)}{P(t)}\right)-A(t)(\gamma+\theta+\delta)+\psi \mathrm{M}(t)$

Where $\mathrm{P}(\mathrm{t})=\mathrm{N}(\mathrm{t})+\mathrm{M}(\mathrm{t})+\mathrm{A}(\mathrm{t})$ and $\mathrm{N}>0, \mathrm{M}>0$ and $\mathrm{A}>0$

All variables and parameters in the model (1) are considered to be positive, and the model lies in the region $G=(N ; M ; A) C R_{+}{ }^{3}: P \leq$ $\mu / \delta$.

\subsection{Basic properties}

\subsubsection{Invariant region}

The study shows that the solutions are bounded in the invariant region $\mathrm{GD}$, where $\mathrm{GD}=(\mathrm{N} ; \mathrm{M} ; \mathrm{A}): \mathrm{P} \leq \mu / \delta$

Theorem 1: The solutions of the model are contained in the feasible region $G$.

Proof. Adding the system of three equations of (3.1) we have

$\frac{d P}{d t}=\mu-\delta P$

Solving equation (3) using $\mathrm{e}^{\delta \mathrm{t}}$ as an integrating factor we obtain

$P(t)=\frac{\mu}{\delta}+C e^{-\delta t}$

At $\mathrm{t}=0$ 
$P(0)=\frac{\mu}{\delta} \leq C$
$P \leq \frac{\mu}{\delta}+\left(P(0)-\frac{\mu}{\delta}\right) e^{-\delta t}$

Where $\mathrm{P}(0)$ is the initial population; which implies that

$0 \leq P \leq \frac{\mu}{\delta}$

Therefore the solutions are bounded in the invariant region

$\omega=N(t), M(t), A(t) \in \mathrm{R}_{+}^{3}: N(t)+M(t)+A(t) \leq 0$.

\subsubsection{Positivity of solutions}

Since the model monitors a population we assume that all associated parameters are non-negative at all times for all $t \geq 0$. We show that all state variables are non-negative and that all the solutions with positive initial data will remain positive for $\mathrm{t} \geq 0$.

Theorem 2 Let the initial conditions be $\mathrm{N}(0) \geq 0, \mathrm{M}(0) \geq 0$, and $\mathrm{A}(0) \geq 0 \in \mathrm{G}$, then the solutions $\mathrm{N}(\mathrm{t}), \mathrm{M}(\mathrm{t})$ and $\mathrm{A}(\mathrm{t})$ of system $(3.1)$ are positive for all $\mathrm{t} \geq 0$.

Proof.

From the first equation of (1)

$\frac{d N}{d t}=\mu-\Omega N-\phi N-\alpha N\left(\frac{M+A}{P}\right)-\delta N+\theta A$

$\frac{d N}{d t} \geq-\Omega N-\phi N-\alpha N\left(\frac{M+A}{P}\right)-\delta N$

By separating variables and integrating both sides gives

$N(t) \geq e^{-\left[\Omega+\phi+\alpha\left(\frac{M+A}{P}\right)+\delta\right] t} \cdot e^{c_{1}}$

Taking $\mathrm{e}^{\mathrm{C} 1}$ to be $\mathrm{K}$ we have

$N(t) \geq \mathrm{K} e^{-\left[\Omega+\phi+\alpha\left(\frac{M+A}{P}\right)+\delta\right] t}$

Using the initial condition $\mathrm{t}=0$ we have $\mathrm{N}(0)=\mathrm{K}$ which implies that

$N(t) \geq \mathrm{N}(0) e^{-\left[\Omega+\phi+\alpha\left(\frac{M+A}{P}\right)+\delta\right] t}$

Therefore $\mathrm{N}(\mathrm{t}) \geq 0$ for all $\mathrm{t} \geq 0$

From the second equation of (1)

$\frac{d M}{d t}=q \Omega \mathrm{N}+r \phi \mathrm{N}+s \alpha \mathrm{N}\left(\frac{M+A}{P}\right)-M(\mu+\delta)+\gamma \mathrm{A}$

$\frac{d M}{d t} \geq-M(\mu+\delta)$

We integrate by separation of variables

$M(t) \geq \mathrm{K}_{1} e^{-[\mu+\delta] t}$

Where $\mathrm{K}_{1}=e^{c_{1}}$

At $\mathrm{t}=0$

We have $\mathrm{M}(0)=\mathrm{K}_{1}$

Hence $M(t) \geq \mathrm{M}(0) e^{-[\mu+\delta}$

Which implies that $\mathrm{M}(\mathrm{t}) \geq 0$ for all $\mathrm{t} \geq 0$

From the third equation of (1)

$\frac{d A}{d t}=(1-q) \Omega \mathrm{N}+(1-\mathrm{r}) \phi \mathrm{N}+(1-s) \alpha N\left(\frac{M+A}{P}\right)-A(\gamma+\theta+\delta)+\psi M$

$\frac{d A}{d t} \geq-A(\gamma+\theta+\delta)$ 
Integration by separation of variables gives

$A(t) \geq e^{-(\gamma+\theta+\delta) t} \cdot e^{C_{3}}$

Let $e^{c_{3}}$ be $\mathrm{K}_{2}$, therefore

$A(t) \geq \mathrm{K}_{2} e^{-(\gamma+\theta+\delta) t}$

At $\mathrm{t}=0$, we have $\mathrm{A}(0)=\mathrm{K}_{2}$

Hence $A(t) \geq \mathrm{A}(0) e^{-(\gamma+\theta+\delta) t}$

Therefore $A(t) \geq 0$ for all $t \geq 0$.

All state variables are positive for all time $t$. The solutions are therefore non-negative for all $t \geq 0$ and are bounded in the invariant region

$\mathrm{G}$ and thus the model is mathematically well posed and biologically meaningful in the feasible region G.

\section{Model analysis}

\subsection{Stability analysis of equilibrium points}

[13] Defines an equilibrium point as a constant solution of a model system. The equilibrium points of a model system are obtained by setting the right hand side of the differential equations to zero and solving each to get a constant solution. Stability analysis of the model is done to determine the conditions for the spread of the awareness on registration requirements and importance of IDs in a population of those attaining 18 years. This study has two equilibrium points namely, Awareness Free Equilibrium (E0) and Maximum Awareness Equi$\operatorname{librium}\left(\mathrm{M}_{\mathrm{a}}{ }^{*}\right)$

\subsection{Awareness free equilibrium $\left(\mathbf{E}_{0}\right)$}

This is the point where there is no awareness about registration requirements and importance of IDs in the entire population of those attaining 18 years, i.e. when $\mathrm{M}=\mathrm{A}=0$.

Considering system (1) when there is no awareness we get

$$
\begin{aligned}
& \frac{d N}{d t}=\mu-\Omega \mathrm{N}-\phi \mathrm{N}-\delta \mathrm{N} \\
& \frac{d M}{d t}=0 \\
& \frac{d A}{d t}=0
\end{aligned}
$$

Solving the first equation

$$
\begin{aligned}
& N=\frac{\mu}{(\Omega+\phi+\delta)} \\
& E_{0}=\left(\frac{\mu}{(\Omega+\phi+\delta)}, 0,0\right)
\end{aligned}
$$

\subsection{Basic reproduction number $(\mathbf{R O})$}

[14] Defined the Basic Reproduction Number as the average number of secondary infections caused by an infectious individual during its period of infection. In this study, a secondary infection is treated as secondary awareness acquired by an individual on registration requirements and importance of an identity card. The Basic reproduction number measures the potential spread of awareness within the population. If $\mathrm{R}_{0}<1$, then each aware individual in his entire life time will produce less than one aware individual on average hence the awareness on registration of persons will not spread. On the other hand when $\mathrm{R}_{0}>1$, then every aware individual in his entire life time will cause more than one aware individual on average hence awareness information on IDs will invade the population. In this study we seek for $\mathrm{R}_{0}>1$ so that those attaining 18 years in the Kenyan population will be fully sensitized. In this study we use the Next Generation Matrix to derive $\mathrm{R}_{0}$ as presented by [15].

Consider the Next generation matrix $G$ made up of matrices $F$ and $V$, such that

$$
G=F V^{-1}
$$

$$
F=\frac{\delta f_{i}}{\delta x_{j}}\left(x_{0}\right)
$$

$$
V=\frac{\delta v_{i}}{\delta x_{j}}\left(x_{0}\right)
$$


Where by $\mathrm{x}_{0}$ is AFE point, $f_{i}$ is the infection matrix(rate of appearance of new awareness in compartment $\mathrm{i}$ ) and $v_{i}$ is the transition matrix (rate of transfer of individuals from compartment $\mathrm{i}$ by all other means and should be negated). $\mathrm{F}$ is the Jacobian of $f_{i}$ and $\mathrm{V}$ is the Jacobian of $v_{\mathrm{i}}$. The basic reproduction number is given as the dominant eigenvalue or the spectral radius of matrix $\mathrm{G}$. $\mathrm{R}_{0}=\rho \mathrm{FV}{ }^{-1}$. The awareness compartments are $\mathrm{M}$ and $\mathrm{A}$, giving $\mathrm{M}=2$.

We use the second and third equation of system (1) to compute $\mathrm{R}_{0}$

$$
\begin{aligned}
& \frac{d M}{d t}=q \Omega N+r \phi N+s \alpha N\left(\frac{M+A}{P}\right)-M(\psi+\delta)+\gamma A \\
& \frac{d A}{d t}=(1-q) \Omega N+(1-r) \phi N+(1-s) \alpha N\left(\frac{M+A}{P}\right)-A(\gamma+\theta+\delta)+\psi \mathrm{M}
\end{aligned}
$$

Calculating the Jacobian matrix at the awareness free equilibrium gives us

$$
\begin{gathered}
F=\left(\begin{array}{cc}
s \alpha & s \alpha \\
(1-s) \alpha & (1-s) \alpha
\end{array}\right) \\
V=\left(\begin{array}{cc}
\mu+\delta & -\gamma \\
-\psi & \gamma+\theta+\delta
\end{array}\right)
\end{gathered}
$$

Eigen values of $\mathrm{FV}^{-1}$ are

$$
\left(0, \frac{\alpha \gamma+\alpha \delta+\operatorname{s} \alpha \theta+\alpha \psi}{\gamma \delta+\delta^{2}+\delta \theta+\delta \psi+\theta \psi}\right)
$$$$
R_{0}=\rho F V^{-1}
$$

$\mathrm{R}_{0}=\frac{\alpha \gamma+\alpha \delta+\operatorname{s} \alpha \theta+\alpha \psi}{\gamma \delta+\delta^{2}+\delta \theta+\delta \psi+\theta \psi}$

$\mathrm{R}_{0}>1$ if $\alpha>\frac{\gamma \delta+\delta^{2}+\delta \theta+\delta \psi+\theta \psi}{\alpha \gamma+\alpha \delta+s \alpha \theta+\alpha \psi}$

From equation (6) $\mathrm{R}_{0}$ does not depend on awareness rate through Electronic and Print media.

\subsection{Local stability analysis of awareness free equilibrium point $\left(E_{0}\right)$}

To determine the local stability of Awareness Free Equilibrium point, the variation Jacobian Matrix at equilibrium point $\mathrm{JE}_{0}$ of the model system (1) is given by

$$
J E_{0}=\left(\begin{array}{ccc}
-\Omega-\phi-\delta & -\alpha & -\alpha+\theta \\
q \Omega+r \phi & s \alpha-(\psi+\delta) & s \alpha+\gamma \\
(1-q) \Omega+(1-r) \phi & (1-s) \alpha+\psi & (1-s) \alpha-(\gamma+\theta+\delta)
\end{array}\right)
$$

The stability of the awareness free equilibrium point can be analyzed by studying the behavior of $\mathrm{JE}_{0}$. For local stability of awareness free equilibrium all its eigenvalues should have negative real parts. The characteristic function of the matrix with $\lambda$ being the eigenvalues of $\mathrm{JE}_{0}$ is obtained. By using Mathematica software we have the following eigenvalues;

$$
\begin{aligned}
& \lambda_{1}=-\delta \\
& \lambda_{2}=-\frac{1}{2}(\gamma+2 \delta+\theta+\phi+\psi+\Omega-\alpha)+K \\
& \lambda_{3}=-\frac{1}{2}(\gamma+2 \delta+\theta+\phi+\psi+\Omega-\alpha)-K
\end{aligned}
$$

Where

$$
K=\frac{1}{2} \sqrt{w^{2}-4\left(-h \alpha+j \theta+g \phi+z \psi+l \Omega+\mathrm{y} \delta+\delta^{2}\right)}
$$

\section{And}

$w=\gamma+2 \delta+\theta+\phi+\psi+\Omega-\alpha)$ 
$\mathrm{j}=\delta-s \alpha$

$g=\gamma+\delta+r \theta$

$\mathrm{z}=\delta-\alpha+\theta+\phi$

Considering real parts

$\lambda_{1}=-\delta$

$\lambda_{2}=-\frac{1}{2}(\gamma+2 \delta+\theta+\phi+\psi+\Omega-\alpha)$

$\lambda_{3}=-\frac{1}{2}(\gamma+2 \delta+\theta+\phi+\psi+\Omega-\alpha)$

It can be seen that $\mathrm{JE}_{0}$ has negative eigenvalues only when $\mathrm{R}_{0}<1$ and therefore the awareness free equilibrium is locally asymptotically stable.

\subsection{Global stability analysis of awareness free equilibrium point $\left(\mathbf{E}_{\mathbf{0}}\right)$}

In this section, the study analyzes the global stability of awareness free equilibrium by using Castillo-Chavez theorem [16].

We re-write the model system (1) as

$\frac{d X}{d t}=F(X, Z)$

$\frac{d Z}{d t}=G(X, Z)$

$G^{\wedge}(X, 0)$

Where $\mathrm{X}=\mathrm{N}$ and $\mathrm{Z}=(\mathrm{M}, \mathrm{A})$. Here the components of $\mathrm{X} \in \mathrm{R}$ denotes the unaware individuals and $\mathrm{Z} \in \mathrm{R}^{2}$ denote the aware individuals The awareness free equilibrium is now denoted by $\mathrm{E}_{0}=\left(\mathrm{X}^{*}, 0\right)$. To guarantee local asymptotic stability, the following 2 conditions, $(\mathrm{H} 1)$ and $(\mathrm{H} 2)$ must be met.

(H1) $\mathrm{dX} / \mathrm{dt}=\mathrm{F}(\mathrm{X}, 0)$, then $\mathrm{X}^{*}$ is globally asymptotically stable (GAS).

(H2) $G(X, Z)=K Z-G^{\wedge}(X, Z)$, where $G^{\wedge}(X, Z) \geq 0$ for $(X, Z) \in G$, where $K=D_{2} G\left(X^{*}, 0\right)$ is an m-matrix (the off-diagonal elements of $K$ are non-negative) and $G$ is the region where the model makes sense.

If the system (7) satisfies conditions (H1) and (H2) then the following theorem holds;

Theorem 3: The fixed point $E_{0}=\left(X^{*}\right)$, is globally asymptotically stable $(G A S)$ equilibrium of system (7) provided Ro $<1$ is locally asymptotically stable and that the assumptions (H1) and (H2) are satisfied.

Proof

Consider (H1).

Considering the model system (1), we have

$$
\begin{aligned}
& F(X, Z)=\left[\begin{array}{l}
\mu-\Omega N-\phi N-\alpha N\left(\frac{M+A}{P}\right)-\delta N+\theta A \\
q \Omega N+\mathrm{r} \phi N+s \alpha N\left(\frac{M+A}{P}\right)-M(\psi+\delta)+\gamma A \\
(1-q) \Omega N+(1-\mathrm{r}) \phi N+(1-s) \alpha N\left(\frac{M+A}{P}\right)-A(\gamma+\theta+\delta)+\psi M
\end{array}\right] \\
& G(X, Z)=\left[\begin{array}{l}
q \Omega N+\mathrm{r} \phi N+s \alpha N\left(\frac{M+A}{P}\right)-M(\psi+\delta)+\gamma A \\
(1-q) \Omega N+(1-\mathrm{r}) \phi N+(1-s) \alpha N\left(\frac{M+A}{P}\right)-A(\gamma+\theta+\delta)+\psi M
\end{array}\right]
\end{aligned}
$$

Then $\mathrm{X}=\mathrm{N}, \mathrm{Z}=(\mathrm{M}, \mathrm{A})$

Now $F(X, 0)=\left[\begin{array}{l}\mu-\Omega N-\phi N-\delta N \\ 0 \\ 0\end{array}\right]$ 
It is clear that $E_{0}=\left(\frac{\mu}{\Omega+\phi+\delta}, 0,0\right)$ is a GAS of $\frac{d X}{d t}=F(X, 0)$. Hence condition $\left(\mathrm{H}_{1}\right)$ is satisfied.

Now consider $\left(\mathrm{H}_{2}\right)$

$G^{\wedge}(X, Z)=\left[\begin{array}{l}s \alpha(M+A)\left(1-\frac{N}{P}\right)-q \Omega N-\mathrm{r} \phi N \\ (1-s) \alpha(M+A)\left(1-\frac{N}{P}\right)-(1-q) \Omega N-(1-\mathrm{r}) \phi N\end{array}\right]$

Since $\mathrm{q} \leq 1, \mathrm{r} \leq 1, \mathrm{~s} \leq 1$ and $0 \leq(\mathrm{M}+\mathrm{A}) \leq \mathrm{N} \leq \mathrm{P}$, it is clear that $G^{\wedge}(X, Z) \geq 0$.

Then we have

$\mathrm{X}=\mathrm{N}$ and $\mathrm{Z}=(\mathrm{M}, \mathrm{A})$

$K=\left(\begin{array}{cc}s \alpha-(\psi+\delta) & s \alpha+\gamma \\ (1-s) \alpha+\psi & (1-s) \alpha-(\gamma+\theta+\delta)\end{array}\right)$

$Z=\left[\begin{array}{l}M \\ A\end{array}\right]$

$K Z=\left[\begin{array}{l}s \alpha M-(\psi+\delta) M+s \alpha A+\gamma A \\ (1-s) \alpha M+\psi M+(1-s) \alpha M-(\gamma+\theta+\delta) A\end{array}\right]$

$G^{\wedge}(X, Z)=\left[\begin{array}{l}s \alpha(M+A)\left(1-\frac{N}{P}\right)-q \Omega N-\mathrm{r} \phi N \\ (1-s) \alpha(M+A)\left(1-\frac{N}{P}\right)-(1-q) \Omega N-(1-\mathrm{r}) \phi N\end{array}\right]$

Then on substituting the above values we have

$K Z-G^{\wedge}(X, Z)=\left[\begin{array}{l}q \Omega N+\mathrm{r} \phi N+s \alpha N\left(\frac{M+A}{P}\right)-M(\psi+\delta)+\gamma A \\ (1-q) \Omega N+(1-\mathrm{r}) \phi N+(1-s) \alpha N\left(\frac{M+A}{P}\right)-A(\gamma+\theta+\delta)+\psi M\end{array}\right]=G(X, Z)$.

Hence $\left(\mathrm{H}_{2}\right)$ is satisfied. Therefore if $\mathrm{R}_{0}<1$ then the fixed point $\mathrm{E}_{0}$ is locally asymptotically stable.

\subsection{Existence of maximum awareness equilibrium point $\left(\mathrm{Ma}_{\mathrm{a}}{ }^{*}\right)$}

The Maximum Awareness Equilibrium point is obtained when the basic reproduction number is greater than one and therefore the awareness spreads in the population. This study denotes maximum awareness equilibrium of system (1) as $\mathrm{M}_{\mathrm{a}^{*}}=\left(\mathrm{N}^{*}, \mathrm{M}^{*}, \mathrm{~A}^{*}\right)$ and is obtained by setting the right hand side of equations to zero.

$$
\begin{aligned}
& \mu-\Omega N *-\phi N *-\alpha N *\left(\frac{M *+A *}{P}\right)-\delta N *+\theta A^{*}=0 \\
& q \Omega N *+r \phi N *+s \alpha N *\left(\frac{M *+A *}{P}\right)-M *(\psi+\delta)+\gamma A^{*}=0 \\
& (1-q) \Omega N *+(1-r) \phi N *+(1-s) \alpha N *\left(\frac{M *+A *}{P}\right)-A *(\gamma+\theta+\delta)+\psi M *=0
\end{aligned}
$$

For the existence and uniqueness of maximum awareness equilibrium $\mathrm{M}_{\mathrm{a}^{*}}=\left(\mathrm{N}^{*}, \mathrm{M}^{*}, \mathrm{~A}^{*}\right)$, the conditions $\mathrm{N}^{*}>0$, or $\mathrm{M}^{*}>0$ or $\mathrm{A}^{*}>0$, must be satisfied. Solving for $\mathrm{N}^{*}, \mathrm{M}^{*}$ and $\mathrm{A}^{*}$; we get

$$
\begin{aligned}
& N^{*}=\frac{P(A * \theta+\psi)}{A * \alpha+M * \alpha+P \delta+P \phi+P \Omega} \\
& M *=\frac{-A * N * s \alpha-A * P \gamma-N * \operatorname{Pr} \phi-N * P q \Omega}{N * \alpha-P \delta-p \psi} \\
& A^{*}=\frac{M * N * \alpha-M * N * s \alpha+N * \operatorname{P} \phi+N * \operatorname{Pr} \phi+M * P \psi+N * P \Omega-N * P q \Omega}{-N * \alpha+N s \alpha+P \gamma+P \delta+p \theta}
\end{aligned}
$$


Local stability of the maximum awareness equilibrium is analyzed by the use of additive compound matrix approach [17]. If $\mathrm{R}_{0}>1$ then the equation model (8) has a unique maximum awareness equilibrium given by $\mathrm{M}_{\mathrm{a}}{ }^{*}=(\mathrm{N} *, \mathrm{M} *, \mathrm{~A} *)$ in $\mathrm{G}$. Local stability of the maximum awareness equilibrium is determined by the variational matrix $J\left(\mathrm{M}_{\mathrm{a}}^{*}\right)$ of the non-linear system.

$J\left(M_{a}^{*}\right)=\left(\begin{array}{ccc}-\Omega-\phi-\alpha\left(\frac{M^{*}+A^{*}}{P}\right)-\delta & -\alpha \frac{N^{*}}{P} & -\alpha \frac{N^{*}}{P}+\theta \\ q \Omega+r \phi+s \alpha\left(\frac{M^{*}+A^{*}}{P}\right) & s \alpha \frac{N^{*}}{P}-(\psi+\delta) & s \alpha \frac{N^{*}}{P}+\gamma \\ (1-q) \Omega+(1-r) \phi+(1-s) \alpha\left(\frac{M *+A^{*}}{P}\right) & (1-s) \alpha \frac{N^{*}}{P}+\psi & (1-s) \alpha \frac{N^{*}}{P}-(\gamma+\theta+\delta)\end{array}\right)$

Lemma 1: Let $J\left(M_{a^{*}}\right)$ be the variational matrix corresponding to $M_{a^{*}}$. If $\operatorname{tr}\left(J\left(M_{a^{*}}\right)\right)$, $\operatorname{det}\left(J\left(M_{a^{*}}\right)\right)$ and $\operatorname{det}\left(J^{[2]}\left(M_{a^{*}}\right)\right)$ are all negative, then all the eigenvalues of $J\left(M_{a}^{*}\right)$ have negative real parts.

Theorem 4: If $R_{0}>1$, the maximum awareness equilibrium $M_{a}^{*}$ of the model (1) is locally asymptotically stable in $G$.

Proof

From Jacobian matrix $\mathbf{J}\left(\mathrm{M}_{\mathrm{a}}{ }^{*}\right)$ we have

$\operatorname{tr}\left(J\left(M_{a}^{*}\right)\right)=-\frac{\left(M *+A^{*}\right)}{P} \alpha+\frac{N *(1-s) \alpha}{P}+\frac{N * s \alpha}{P}-\gamma-3 \delta-\theta-\phi-\psi-\Omega<0$.

$\operatorname{det}\left(J\left(M_{a}^{*}\right)\right)=-\frac{A * \alpha \gamma \delta}{P}-\frac{M * \alpha \gamma \delta}{P}+\frac{N * \alpha \gamma \delta}{P}-\frac{A * \alpha \delta^{2}}{P}-\frac{M * \alpha \delta^{2}}{P}-\frac{N * \alpha \delta^{2}}{P}-z-\frac{A * s \alpha \theta \delta}{P}+\frac{M * s \alpha \theta \delta}{P}+\frac{N * s \alpha \theta \delta}{P}$

$-l-\frac{A * \alpha \delta \psi}{P}-\frac{M * \alpha \delta \psi}{P}-\frac{N * \alpha \delta \psi}{P}-h<0$

Where

$z=\gamma \delta^{2}+\delta^{3}$

$l=\delta^{2} \theta+\gamma \delta \theta+\delta^{2} \phi+r \delta \theta \phi$

$h=\delta^{2} \psi+\delta \theta \psi+\delta \phi \psi+\gamma \delta \Omega+\delta^{2} \Omega+\delta \theta \Omega+\delta \Omega \psi$

Hence, the trace and determinant of the Jacobian matrix $\mathrm{J}\left(\mathrm{M}_{\mathrm{a}}{ }^{*}\right)$ are all negative. The second additive compound matrix is obtained from the following lemma.

Lemma 2: Let $P$ and $Q$ be subset of $J^{[2]}\left(M a^{*}\right)$. The $(P, Q)$ entry of $N_{i j}\left(J^{[2]}\left(M a^{*}\right)\right)$ is the coefficient of $C$ in the expansion of the determinant of the sub-matrix of $J\left(M_{a^{*}}\right)+C I$ indexed by row in $P$ and column in $Q$.

Proof

The sub-matrix of $\mathbf{J}\left(\mathrm{Ma}^{*}\right)+\mathrm{CI}$ is given as

$\left(\begin{array}{ccc}-\left(\Omega+\phi+\alpha\left(\frac{M^{*}+A^{*}}{P}\right)+\delta\right)+C & -\alpha \frac{N^{*}}{P} & -\alpha \frac{N^{*}}{P}+\theta \\ q \Omega+r \phi+s \alpha\left(\frac{M *+A^{*}}{P}\right) & -\left((\psi+\delta)-s \alpha \frac{N^{*}}{P}\right)+C & s \alpha \frac{N^{*}}{P}+\gamma \\ (1-q) \Omega+(1-r) \phi+(1-s) \alpha\left(\frac{M *+A^{*}}{P}\right) & (1-s) \alpha \frac{N^{*}}{P}+\psi & -\left((\gamma+\theta+\delta)-(1-s) \alpha \frac{N^{*}}{P}\right)+C\end{array}\right)$

The sub-matrix of $\mathrm{J}\left(\mathrm{M}_{\mathrm{a}^{*}}\right)+\mathrm{CI}$ indexed by rows and columns is given by

$$
\left(\begin{array}{cc}
-\left(\Omega+\phi+\alpha\left(\frac{M^{*}+A^{*}}{P}\right)+\delta\right)+C & -\alpha \frac{N^{*}}{P} \\
q \Omega+r \phi+s \alpha\left(\frac{M^{*}+A^{*}}{P}\right) & -\left((\psi+\delta)-s \alpha \frac{N^{*}}{P}\right)+C
\end{array}\right)
$$

The coefficient of $\mathrm{C}$ in the determinant of this matrix is

$$
-\alpha\left(\frac{M *+A *}{P}\right)+s \alpha \frac{N *}{P}-(2 \delta+\phi+\psi+\Omega)
$$

And thus the $(1,1)$ entry of $\mathrm{N}_{\mathrm{ij}}$ is

$-\alpha\left(\frac{M^{*}+A^{*}}{P}\right)+s \alpha \frac{N^{*}}{P}-(2 \delta+\phi+\psi+\Omega)$ 
Other entries are obtained by the same method and $\left(\mathrm{J}^{[2]}\left(\mathrm{M}^{*}\right)\right)$ becomes

$$
\left.\begin{array}{ccc}
-\alpha\left(\frac{M *+A}{P}\right)+s \alpha \frac{N *}{P}-(2 \delta+\phi+\psi+\Omega) & \frac{N * s \alpha}{P}+\gamma & \frac{N * \alpha}{P}-\theta \\
(1-s) \alpha \frac{N *}{P}+\psi & -\alpha\left(\frac{M *+A *}{P}\right)+(1-s) \alpha \frac{N *}{P}-(\gamma+2 \delta+\theta+\phi+\Omega) & -\frac{N * \alpha}{P} \\
-\alpha\left(\frac{M *+A *}{P}\right)+s \alpha\left(\frac{M *+A *}{P}\right)-(\phi+\Omega)+\mathrm{r} \phi+q \Omega & s \alpha\left(\frac{M *+A *}{P}\right)+\mathrm{r} \phi+q \Omega & \alpha \frac{N *}{P}-(\gamma+2 \delta+\theta+\psi)
\end{array}\right)
$$

Thus, according to Lemma 1, the maximum awareness equilibrium $\mathrm{M}_{\mathrm{a}}{ }^{*}$ of the model system (1) is locally asymptotically stable.

\section{Numerical simulations}

Numerical simulations were carried out using mathematica software to illustrate the behavior of the system for different values of the model parameters. Some of the parameters which are compatible with awareness of a new product in the market were obtained from literature while others were estimated.

The parameter values are shown in Table 3

Table 3: Model Parameters and Values

\begin{tabular}{lll}
\hline Parameter & Value & Source \\
\hline$\alpha$ & 0.4 & (Aminiel et al., 2015) \\
$\Omega$ & 0.2 & Estimated \\
$\Phi$ & 0.1 & Estimated \\
$\mathrm{s}$ & 0.6 & (Aminiel et al., 2015) \\
$\mathrm{q}$ & 0.7 & Estimated \\
$\mathrm{r}$ & 0.4 & Estimated \\
$\mu$ & 0.5 & (Aminiel et al., 2015) \\
$\delta$ & 0.001 & Estimated \\
$\theta$ & 0.03 & (Aminiel et al., 2015) \\
$\gamma$ & 0.6 & (Aminiel et al., 2015) \\
$\psi$ & 0.0001 & (Aminiel et al., 2015) \\
\hline
\end{tabular}

\subsection{Numerical illustration of stability points of the model}

To illustrate the awareness free equilibrium and maximum awareness equilibrium, numerical simulations of $\mathrm{R}_{0}<1$ and $\mathrm{R}_{0}>1$ are shown below in Figure 2 and 3 respectively with values of $\alpha$ (awareness rate through the word of mouth) being varied $(\alpha=0.3,0.6$ and 0.9 ).

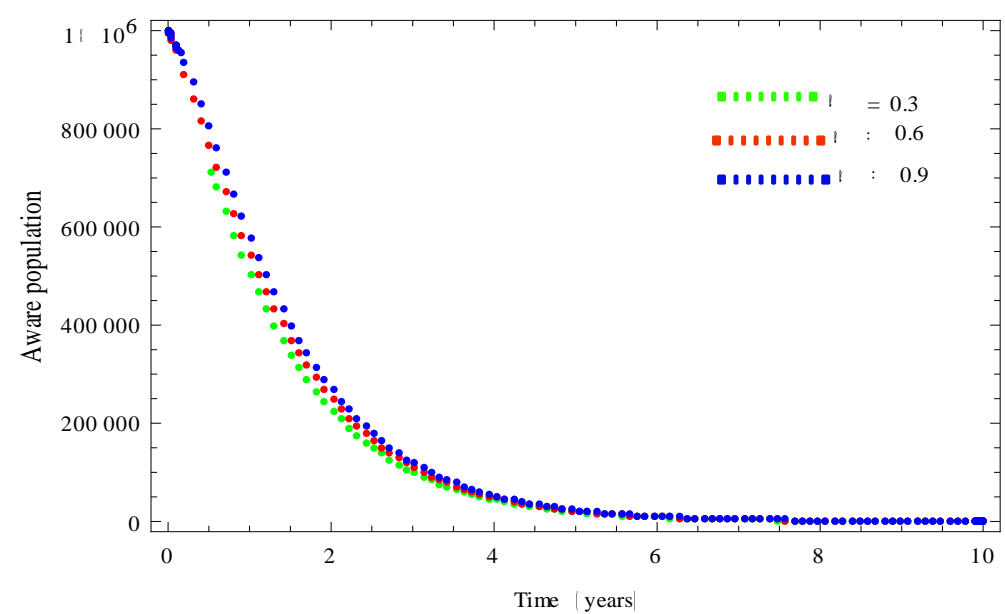

Fig. 2: Numerical Solutions when $\mathrm{R}_{0}<1(\mathrm{~s}=0.00006, \delta=0.8, \theta=0.7, \gamma=0.00006, \psi=0.4)$.

Figure 2 shows that when $\mathrm{R}_{0}<1$, all the trajectories of aware population converges to zero regardless of the values of $\alpha$. Therefore, awareness free equilibrium $\mathrm{E}_{0}$ is asymptotically stable supporting theorem 3. 


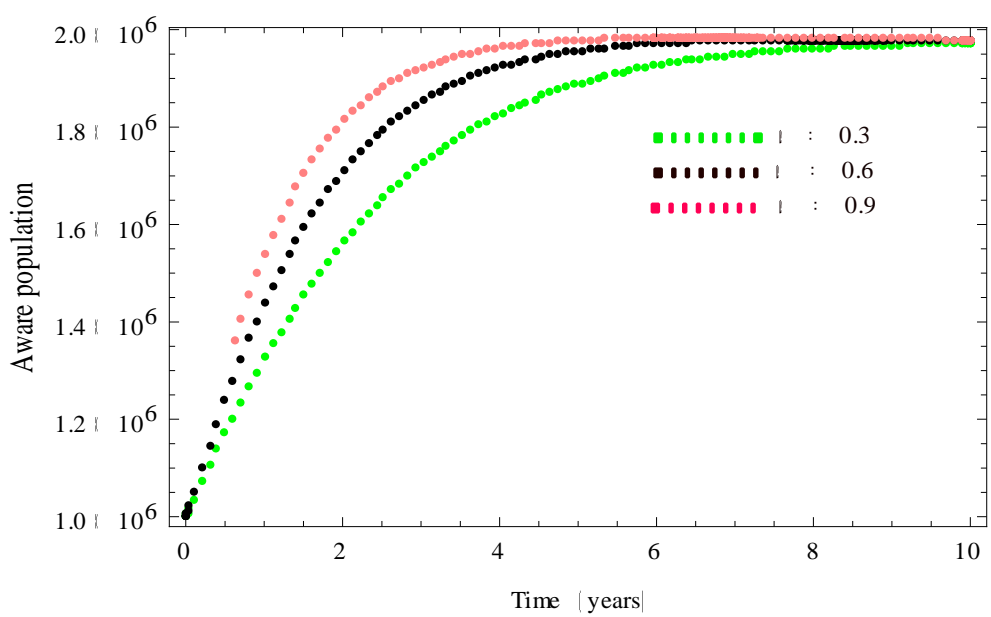

Fig. 3: Numerical Solutions when $\mathrm{R}_{0}>1(\mathrm{~s}=0.8, \delta=0.001, \theta=0.3, \gamma=0.8, \psi=0.0001)$.

Figure 3 shows that when $\mathrm{R}_{0}>1$ all the trajectories of aware population converge at $\left(\mathrm{M}_{\mathrm{a}}{ }^{*}\right)$ regardless of the values of $\alpha$. Therefore the maximum awareness equilibrium is locally asymptotically stable supporting theorem 4

\section{Conclusion}

In this paper, a mathematical model based on a system of Ordinary Differential Equations to model public sensitization initiatives on registration requirements and importance of ID cards in Kenya is developed. The model is composed of the following compartments: Unaware, Aware-Adopters and Aware-Non-Adopters. Computation of basic reproduction number $\mathrm{R}_{0}$ which is the threshold parameter, was done using the next generation matrix approach and showed that if $\mathrm{R}_{0}<1$ the awareness on Registration requirements and importance of IDs does not spread, and when $\mathrm{R}_{0}>1$ the awareness spreads. Stability analysis of awareness free equilibrium exists and it is locally and globally asymptotically stable when the basic reproduction number is less than one. Analysis of the Maximum awareness equilibrium shows its existence when basic reproduction number is greater than one. Maximum awareness equilibrium is also locally asymptotically stable. This means keeping $\mathrm{R}_{0}$ greater than one is a possible way of increasing awareness on registration of persons. Numerical simulation is done by varying the parameter $\alpha$ (awareness rate through word of mouth) to show the equilibrium points when $\mathrm{R}_{0}<1$ and $\mathrm{R}_{0}>1$.

\section{Acknowledgement}

The authors would like to thank Chuka University for the internal research funds granted.

\section{References}

[1] G. Olivier. Metadata for identity management of population registers. Future Internet, 3:130-143, 2011. doi: FutureInternet2011,3,130-143; https://doi.org/10.3390/fi3020130.

[2] KNCHR. An Identity Crisis? A Study of Issuance of National Identity Cards in Kenya. Technical report, KNCHR, Nairobi, 2007.

[3] CAJ. An Investigation Report on the Crisis of Acquiring Identification Documents in Kenya. Technical report, Nairobi, 2015.

[4] N. O. Onyango. Factors Influencing Timely Registration of Persons in Kisumu City, Kenya. Master's thesis, UON, Nairobi, 2014.

[5] M. Gacheri. Strategy Implementation Practices in the Department of National Registration Bureau in Kenya. Master's thesis, UoN, Nairobi, 2012.

[6] Kenya National Assembly. Report of the Joint Committee on Administration and National Security; and Defence and Foreign Relations, on the Inquiry into the Westgate Mall Terror Attack, and other terrorist Attacks in Mandera in North Eastern and Kilifi in the Coastal Region. Technical report, Nairobi, 2013.

[7] R. Ullah, G. Zaman, and S. Islam. Stability Analysis of a General SIR Epidemic Model. VFAST Transactions on Mathematics, 1:16-20, 2013. doi: http://vfast.org/index.php/VTM@2013ISSN:2309-0022.

[8] N. Kaur, M. Ghosh, and S. S. Bhatia. Modeling and Analysis of an SIRS Epidemic Model with Effect of Awareness Programs by Media. Mathematical and Computational Sciences, 8(1):233-239, 2014. https://doi.org/10.1155/2014/680743.

[9] O. G. Agaba, N. Y. Kyrychko, and B. K. Blyuss. Mathematical Model for the Impact of Awareness on Dynamics of Infectious Diseases. Mathematical Bio Sciences, 286: 22-30, 2017. https://doi.org/10.1016/j.mbs.2017.01.009.

[10] P. K. Roy, S. Saha, and F. Al Basir. Effect of Awareness Programs in Controlling the Disease HIV/AIDS: an Optimal Control Theoretic Approach. Advances in Difference Eguation, 2015:217, 2015. https://doi.org/10.1186/s13662-015-0549-9.

[11] H. Huo and Q. Wang. Modelling the Influence of Awareness Programs by Media on the Drinking Dynamics. Abstract and Applied Analysis, 2014:18, 2014. https://doi.org/10.1155/2014/938080.

[12] J. Aminiel, D. Kajunguri, and E. A. Mpolya. Mathematical Modeling on the Spread of Awareness Information to Infant Vaccination. Applied Mathematics, 5(6):101-110, 2015.

[13] M. B. Onyuma. Modelling Cholera Transmission Incorporating Media Coverage. Master's thesis, Moi University, Eldoret, 2017.

[14] O. Diekmann, J. A. P. Heesterbeek, and J. A. J. Metz. On the Definition and the Computation of the Basic Reproduction Ratio R0 in Models for Infectious Diseases in Heterogeneous Populations. Mathematical Biology, 28(4):365-382, 1990. https://doi.org/10.1007/BF00178324.

[15] P. Van Den Driessche and J. Watmough. Reproduction Numbers and Threshhold Endemic Equilibria for Compartmental Models of Disease Transmission. Mathematical Biosciences, 180(1-2):29-48, 2002. https://doi.org/10.1016/S0025-5564(02)00108-6.

[16] C. Castillo-Chavez, Z. Feng, and W. Huang. On the computation of R0 and its role on global stability. Springer-Verlag, $125: 229-250,2002$. https://doi.org/10.1007/978-1-4757-3667-0_13.

[17] N. M. Laurencia, S. M. Estomih, and D. M. Oluwole. Temporal Model for Dengue Disease with Treatment. Advances in Infectious Diseases, 5:2136, 2015. https://doi.org/10.4236/aid.2015.51003. 